\title{
ORIGINAL
}

\section{RESULTADOS COMUNICADOS POR EL PACIENTE EN LAS FICHAS TÉCNICAS DE MEDICAMENTOS APROBADOS EN ESPAÑA DURANTE EL PERÍODO 2000-2012}

\author{
José Ignacio Pueyo Velasco (1) y Javier Rejas Gutierrez (2) .
}

(1) Agencia Española de Medicamentos y Productos Sanitarios. Madrid. España.

(2) Health Economics and Outcomes Research. Pfizer SLU. Alcobendas. Madrid. España.

Sin conflicto de intereses

\section{RESUMEN}

Fundamentos: El término Patient Reported Outcomes (PRO) es definido como el resultado de la evaluación de un medicamento por el paciente y basada en su percepción de la enfermedad y su(s) tratamientos(s) El objetivo del estudio es determinar la utilización de PRO y compararla con otros tipos de variables de la eficacia contenidas en las fichas técnicas (FT) de medicamentos.

Métodos: Revisión y análisis descriptivo de las variables de eficacia utilizadas en los ensayos clínicos de principios activos aprobados en España durante el período 2000-2012, descritas en las fichas técnicas clasificándolas en las siguientes categorías: PRO, resultados informados por el clínico (CRO) y resultados finales basados en pruebas de laboratorio (ML). Para las comparaciones se realizaron la prueba $\mathrm{chi}^{2}$ o la prueba exacta de Fisher, y se calcularon odd ratios para valorar la intensidad de la asociación

Resultados: De las 330 FT estudiadas se incluyeron PROs en 65 $(19,7 \%)$, los CRO en $188(57 \%)$ y los ML en $128(38,7 \%)$. Los PRO se utilizaron como único resultado final de eficacia en 6 medicamentos. Se observó una tendencia creciente de la significación estadística en la utilización de PRO a lo largo del tiempo estudiado $(\mathrm{R} 2=0,525 ; \mathrm{p}=0,079)$.

Conclusiones: La frecuencia de utilización de PRO en FT es claramente inferior a la de las otras medidas de la eficacia, siendo la diferencia significativamente mayor en el caso de su inclusión como variable única final. La utilización de PROs fue más frecuente en medicamentos del sistema nervioso, agentes inmunomoduladores, sistema respiratorio y sistema musculoesquelético.

Palabras clave: Evaluación del resultado de la atención al paciente. Etiquetado de medicamentos. Aprobación de drogas. Calidad de vida.

\section{Correspondencia}

José Ignacio Pueyo Velasco

Agencia Española de Medicamentos y Productos Sanitarios

Madrid, España

$\mathrm{C} /$ Campezo ${ }^{\circ} 1$, Edificio 8

28022 Madrid

jipueyo@aemps.es

\section{ABSTRACT}

\section{Review of Patient Reported Outcomes in the Summary of the Product Characte- ristics of Medicines Approved in Spain}

Background: The term "Patient Reported Outcomes" (PRO) is defined as any result assessed directly by the patient and based on his perception of the illness and its treatment. The scope of the study is to determine and compare the use of PRO measures with other types of effectiveness endpoints in the summary of the products characteristics (SPC) of medicines with active substances approved by first time from 2000 to 2012 in Spain.

Methods: A review and descriptive analysis of the effectiveness endpoints used in the clinical trials described in the SPCs studied, classifying them into the following categories: PRO, clinician-reported outcomes (CROs), and laboratory test/device measurement endpoints (ML). Chisquare and Fisher exact texts were used for comparative purposes, and odds ratio to determine the strongest of association.

Results: PROs were included in $65(19.7 \%)$ of the SPCs studied. CRO were the most frequently reported $188(57 \%)$ cases, followed by ML: 128 $(38,7 \%)$. PROs were included as only effectiveness endpoint in 6 medicines. A growing trend toward statistical significance $(R 2=0.525 ; \mathrm{p}=0.079)$ has been observed in reporting PROs during the period of time studied.

Conclusion: The use frequency of PRO in SPCs is clearly minor compared to other efficacy endpoints considered, being the difference significantly higher in case of its use as only efficacy endpoint. The therapeutic areas where PROs were most reported were nervous system medicines, immunologic system modulator medicines, respiratory system, muscle and skeleton system.

Keyword: Patient outcome assessment. Drug labeling. Drug approval. Quality of life. 


\section{INTRODUCCIÓN}

La información proporcionada por el paciente sobre su estado de salud es habitualmente utilizada en la práctica clínica, aunque no ha sido hasta los últimos años cuando se ha estandarizado y se ha incluido el término "resultados comunicados por el paciente" en la terminología clínica. El término Patient Reported Outcomes (PRO), o Resultados Comunicados por el Paciente, es definido como todo resultado evaluado directamente por el propio paciente y basado en su percepción de la enfermedad y su(s) tratamiento(s). Se puede generar evidencia de la efectividad de un tratamiento desde la perspectiva del paciente utilizando medidas de resultados comunicados por el paciente [Patient Reported Outcomes Measures (PROMs)]. Se propone PRO como término general que comprende tanto valoraciones unidimensionales como multidimensionales de síntomas, calidad de vida relacionada con la salud (CVRS), estado de salud, cumplimiento terapéutico y satisfacción con el tratamiento ${ }^{1}$. El concepto de calidad de vida relacionada con la salud es un tipo de PRO que incluye específicamente no solo resultados percibidos por el paciente sino evaluaciones más amplias sobre el impacto de su enfermedad en su actividad normal y bienestar.

Debido al incremento de la utilización de PRO en investigación clínica, las agencias reguladoras los han tenido en cuenta dentro del proceso de regulación de los medicamentos publicando documentos sobre este tema. En 2004, el Comité de Medicamentos para Uso Humano (CHMP) de la Agencia Europea de Medicamentos (EMA) publicó el documento Reflection Paper on the Regulatory Guidance for the Use of Health-Related Quality of Life Measures in the Evaluation of Medicinal Products ${ }^{1}$, cuyo objetivo es analizar el lugar que puede tener la calidad de vida relacionada con la salud
(CVRS), un tipo específico de PRO, en la evaluación del medicamento. En este documento la EMA sostiene el interés que tiene la CVRS en enfermedades severas que ponen en peligro la vida, como el cáncer, donde la CVRS puede proporcionar información importante siempre que el beneficio en CVRS no suponga una reducción de la eficacia. En enfermedades crónicas que no suponen un riesgo para la vida, la información sobre CVRS puede ser importante para la elección de un medicamento sobre otro en la práctica clínica cuando tienen perfiles de seguridad y eficacia similares. Y en enfermedades crónicas con episodios de exacerbación, donde la evaluación de CVRS también puede ser de interés ${ }^{2}$. En 2006, la Food and Drug Administration (FDA) norteamericana publicó un borrador de guía para la industria Guidance for industry: patient-reported outcomes measures: use in medical product development to support labeling claims $^{3}$ con el objetivo de describir cómo la FDA evalúa los instrumentos de medición de PRO utilizados como resultados finales en ensayos clínicos y cómo utilizar los datos obtenidos para describir su efectividad en el etiquetado. Esta guía fue actualizada en el año $2009^{4}$.

De acuerdo con la guía, los instrumentos de medición de PRO se utilizan en los ensayos clínicos de medicamentos nuevos por alguna de las siguientes razones:

- Algunos de los efectos de los tratamientos, como el alivio del dolor, sólo son conocidos por el paciente, ya que no hay medidas observables o físicas relacionadas con el resultado medido.

- Los pacientes proporcionan una perspectiva única de la efectividad del tratamiento. Mediante instrumentos de medición de PRO se puede conocer qué quieren y qué esperan los pacientes de un determinado tratamiento y qué resultado es realmente importante para ellos, ya que 
una mejora de resultados clínicos no corresponde necesariamente a mejoras en la actividad del paciente o en cómo se siente.

Las evaluaciones formales pueden ser más fiables que las entrevistas informales. Los instrumentos de medición de PRO correctamente desarrollados y validados muestran resultados que concuerdan con los resultados obtenidos por expertos y son especialmente útiles en estudios con múltiples investigadores.

Ambas guías tienen puntos en común pero también muestran diferencias. No solo reconocen la utilidad de los resultados percibidos por el paciente para la evaluación de resultados en salud sino que también desarrollan aspectos metodológicos tales como el diseño del estudio, el análisis estadístico, los test de hipótesis así como la fiabilidad y la validez de los resultados obtenidos mediante instrumentos de medición de PRO. Sin embargo, mientras que la FDA reconoce que todos los PRO, incluida la CVRS, se pueden utilizar como resultado final de la eficacia en ensayos clínicos, especialmente para tratamientos crónicos, la EMA no le otorga la misma importancia por el momento a todas las medidas de PRO. Mientras que los síntomas percibidos por el paciente, tales como el dolor, valorados por ellos mismos, son bien aceptados como resultados primarios o secundarios en ensayos clínicos utilizados en el registro del medicamento, la evaluación de la CVRS se refiere como un resultado opcional teniendo en cuenta que la base para la aprobación de un nuevo medicamento es su eficacia y seguridad clínica en una condición dada $^{5}$.

La Organización Mundial de la Salud (OMS) declaró que la implicación del paciente en el cuidado de su salud no es solo deseable sino también una necesidad social, económica y técnica ${ }^{6,7}$. Los pacientes quieren involucrarse en el proceso de decisión, especialmente cuando existen varias alternativas terapéuticas ${ }^{8}$. Los pacientes tienen la última responsabilidad en la toma de decisiones relacionadas con su salud. Específicamente, el paciente decide cuando visitar al médico, si sigue o no el consejo médico que le han proporcionado y en último término si cumple o no con la prescripción de un medicamento o busca un tratamiento alternativo. Consecuentemente, el punto de vista del paciente en relación con los resultados en salud debe de ser tomado más en serio por parte de los diferentes agentes sanitarios ${ }^{9}$.

Por otra parte, el papel fundamental del pagador en el acceso al mercado de nuevos medicamentos, junto con una mayor demanda de información por parte de pacientes sobre la eficacia y efectividad de los nuevos tratamientos, ha incrementado notablemente la competitividad del mercado farmacéutico. Ante este cambio de escenario, la investigación farmacéutica debe generar nuevas proposiciones de valor más allá de los tradicionales valores de seguridad y eficacia clínica. Una forma de conseguirlo es incorporar la perspectiva del paciente sobre el tratamiento que recibe. Se puede generar evidencia de la efectividad de un tratamiento desde la perspectiva del paciente utilizando medidas PRO (PROMs por sus siglas en inglés $)^{10}$, debidamente validadas en la cultura en la que se usan, que permiten medir aspectos determinados, como síntomas, limitaciones en la actividad diaria, CVRS, etcétera.

Es destacable el hecho de que suele haber discrepancias entre el punto de vista del clínico y el paciente sobre la efectividad de un tratamiento ${ }^{11-13}$. Los clínicos suelen informar menos problemas que los pacientes, pueden subestimar la severidad del problema y sobreestimar la mejora con el tratamiento ${ }^{14-17}$. 
La ficha técnica o resumen de las características de un medicamento sintetiza la información científica esencial para los profesionales sanitarios reflejando las condiciones de uso autorizadas para el mismo. Las autoridades sanitarias aprueban la ficha técnica en la que constan las indicaciones terapéuticas para las que el medicamento ha sido autorizado de acuerdo con los estudios que avalan su autorización.

El objetivo de este estudio es conocer los tipos de resultados de eficacia utilizados en las fichas técnicas de los medicamentos y comprobar en qué grado los PROs son utilizados en el procedimiento regulatorio de aprobación de medicamentos en España así como describir la utilización de PROs según grupo terapéutico e indicación en las fichas técnicas de los medicamentos objeto de estudio.

\section{MATERIAL Y MÉTODOS}

Se realizó una revisión de las fichas técnicas de los medicamentos aprobados por primera vez en España desde enero de 2000 hasta diciembre de 2012. Se excluyeron las moléculas aprobadas anteriormente bajo otra denominación comercial, los medicamentos genéricos y los diferentes formatos o formas farmacéuticas de una misma molécula. Inicialmente se realizó una búsqueda en la base de datos del Ministerio de Sanidad RAEFAR (aplicación informática de registro de medicamentos de uso humano) con los siguientes parámetros de búsqueda: clase de solicitud igual "completa/independiente nuevo active pharmaceutical ingredient (API)" y tipo de especialidad distinto a "medicamento genérico". Al realizar esta primera búsqueda no se obtuvieron registros en el año 2006 de solicitudes de nuevas moléculas, por lo que se realizó una búsqueda adicional con el parámetro de búsqueda: clase de solicitud igual a "desconocido" y cotejando los resultados con la base de datos del Centro de Información online de Medicamentos (CIMA), disponible en la página web de la Agencia Española de Medicamentos y Productos Sanitarios, con el fin de asegurar que las moléculas hubieran sido aprobadas por primera vez en España en el período de tiempo objeto del estudio. Debido a este hecho, los resultados recogidos en el estudio quedan ampliados a moléculas aprobadas por primera vez en España en el período mencionado y no a la aprobación de moléculas nuevas, que quedan definidas en la vigente normativa como moléculas aprobadas por primera vez en Europa. Es decir, el estudio recoge tanto moléculas nuevas como moléculas que aunque hubieran sido aprobadas anteriormente en algún país de la Unión Europea fueran registradas por primera vez en España durante el período 2000-2012.

La fuente primaria de obtención de los datos finales de eficacia fue el punto 5.1 "Propiedades farmacodinámicas" de las fichas técnicas aprobadas de los medicamentos. En el caso de medicamentos retirados se utilizó la última versión disponible de la ficha técnica. En el caso de varias indicaciones en un medicamento se consideraron todas sus indicaciones juntas. Las variables finales de eficacia se clasificaron siguiendo el artículo de Willke RJ y colaboradores ${ }^{18}$ de acuerdo a tres categorías y se registró su ausencia o presencia en cada uno de los medicamentos revisados. Los medicamentos podían presentar uno, dos o los tres tipos de variables finales de eficacia: Pruebas de laboratorio y dispositivos de medida (ML), resultados informados por el médico (CRO) y/o resultados comunicados por el paciente (PRO). Las medidas realizadas mediante pruebas de laboratorio y dispositivos incluyeron dimensiones numéricas objetivas normalmente realizadas por equipos o dispositivos. Los resultados informados por el médico incluyeron aquellos que se pueden considerar resultados tradiciona- 
les de eficacia que son observados por el clínico (cura de infección o ausencia de lesión) o bien requieren su interpretación (resultados radiológicos y respuesta tumoral). Además, los resultados informados por el médico pueden suponer la utilización de escalas formales o no formales que completa utilizando información sobre el paciente. Los CRO que requieren información del paciente se distinguen de los PRO en que la información recogida precisa de la valoración o interpretación del médico cuando se realiza el formulario, mientras que en los PRO se recoge sin modificar la información que ha proporcionado directamente el paciente en respuesta a preguntas previamente establecidas. Por último, las variables finales de eficacia que se clasifican como PRO incluyen medidas formales de calidad de vida relacionada con la salud y cualquier otro resultado final que esté basado de modo principal en información proporcionada por el paciente. Los PROs que se clasifican como escalas formales son cuestionarios multi item que tienen un formato estandarizado bien definido, procedimientos de administración y puntuación bien documentados, validez y fiabilidad demostradas y guías para la interpretación de los resultados ${ }^{19}$. Otros PROs incluyen escalas no formales de síntomas, evaluaciones globales del paciente o escalas visuales analógicas, así como resultados comunicados por el paciente mediante registro de eventos (por ejemplo cuadernos de paciente). Se consideraron todos los resultados de eficacia sin clasificarlos en resultados primarios y secundarios. Además, no se tuvieron en cuenta eventos comunicados por el paciente así como cualquier otra información recogida en la sección 4.8 "Reacciones Adversas", ya que el objetivo del estudio se centró en las medidas de beneficio de los medicamentos considerados. Otros datos recogidos utilizados en el análisis fueron el año de aprobación y el grupo farmacoterapéutico.
Se consultaron las páginas web http://www.proqolid.org (Patient Reported Outcome and Quality of Life Instruments Database) y http://www.bibliopro.org (Biblioteca de Patient Reported Outcomes (PRO) en español) para obtener información sobre las características de las escalas formales identificadas en las fichas técnicas.

La base de datos del Ministerio de Sanidad RAEFAR no es de acceso público y se utilizó para aplicar los filtros descritos y obtener el listado de medicamentos objetivo del estudio. Las fichas técnicas de los medicamentos y la información utilizada en el estudio se obtuvieron mediante la base de datos CIMA de libre acceso. La extracción de datos la realizó el único autor con acceso a RAEFAR debido a su trabajo en la Agencia Española de Medicamentos y Productos Sanitarios.

En el estudio se cuantificó la utilización de PROs por área terapéutica y también se identificó y contabilizó la utilización de escalas formales de medición de PROs. El análisis estadístico incluyó un análisis descriptivo con determinación de frecuencias absolutas y la proporción de los distintos resultados analizados según tipo (CRO, PROs, ML) y categorías dentro de los PROs (calidad de vida, síntomas, etc.) y combinaciones de resultados incluidos en las fichas técnicas de medicamentos aprobados, calculándose el intervalo de confianza del $95 \%$ para la proporción de resultados cuando se incluyó un solo tipo de resultados en la ficha técnica de modo que se pudiera realizar una estimación de la magnitud de las diferencias. Las comparaciones de frecuencias entre grupos se realizaron mediante la prueba de la $\mathrm{chi}^{2} \mathrm{o}$ la prueba exacta de Fisher, dependiendo del número de efectivos observados. La intensidad de la asociación en las comparaciones por pares se realizó calculando los correspondientes odds ratios y su intervalo de confianza del $95 \%$. La pro- 
porción de medicamentos aprobados que incluyeron medidas de PROs a lo largo del período de estudio se analizó mediante un análisis simple de tendencia lineal (regresión lineal con el número de medicamentos aprobados en el año como covariable), calculándose el estadístico R2 como medida de ajuste del modelo. Los cálculos estadísticos se realizaron con el paquete estadístico IBM SPSS v17.02020.

\section{RESULTADOS}

Se incluyeron 330 moléculas aprobadas por primera vez en España durante el período 2000-2012. En el estudio se incluyeron todos los medicamentos que cumplían con los parámetros establecidos, si bien algunos mostraron información incompleta tal como la no descripción del resultado final de eficacia en 42 medicamentos, o la no disponibilidad de ficha técnica en 3 medicamentos. Estos casos en los que no constaba la descripción del resultado final de eficacia supusieron el 13,6\% de los medicamentos estudiados, siendo muchos medicamentos de radiodiagnóstico, los cuales, aunque por su indicación terapéutica no resultan de interés para el objetivo del estudio se contabilizaron para la suma total de medicamentos apro- bados. En el caso de los medicamentos Embrel indicados para la artritis reumatoide (AR) y artritis psoriásica (AP), Kineret para la indicación de AR, Humira para la indicación de artritis idiopática juvenil poliarticular (AIJP) y AR, Orencia para la indicación de AR y AIJP, Simponi para las indicaciones de AR y AP, Roactemra para las indicaciones de AR y AIJP, y Cimzia para la indicación de AR utilizaron como resultado final de eficacia el porcentaje de mejoría usando los criterios de respuesta del Colegio Americano de Reumatología (ACR). Los criterios de respuesta del ACR miden la mejoría en articulaciones inflamadas o sensibles y la mejora en tres de los siguientes cinco parámetros: Reacción en fase aguda (tal como tasa de sedimentación), evaluación del paciente, evaluación del clínico, escala de dolor y cuestionario de discapaci$\mathrm{dad} /$ funcionalidad. Se contabilizaron todos los parámetros que componen el criterio de respuesta de forma individual en la realización del estudio. El medicamento Enbrel para la indicación de AP utiliza además como resultado final de eficacia el porcentaje de mejora en el criterio de respuesta de artritis psoriásica (PsARC). Este criterio incluye: Recuento de articulaciones sensibles, recuento de

Figura 1

Frecuencia de tipo de resultados finales

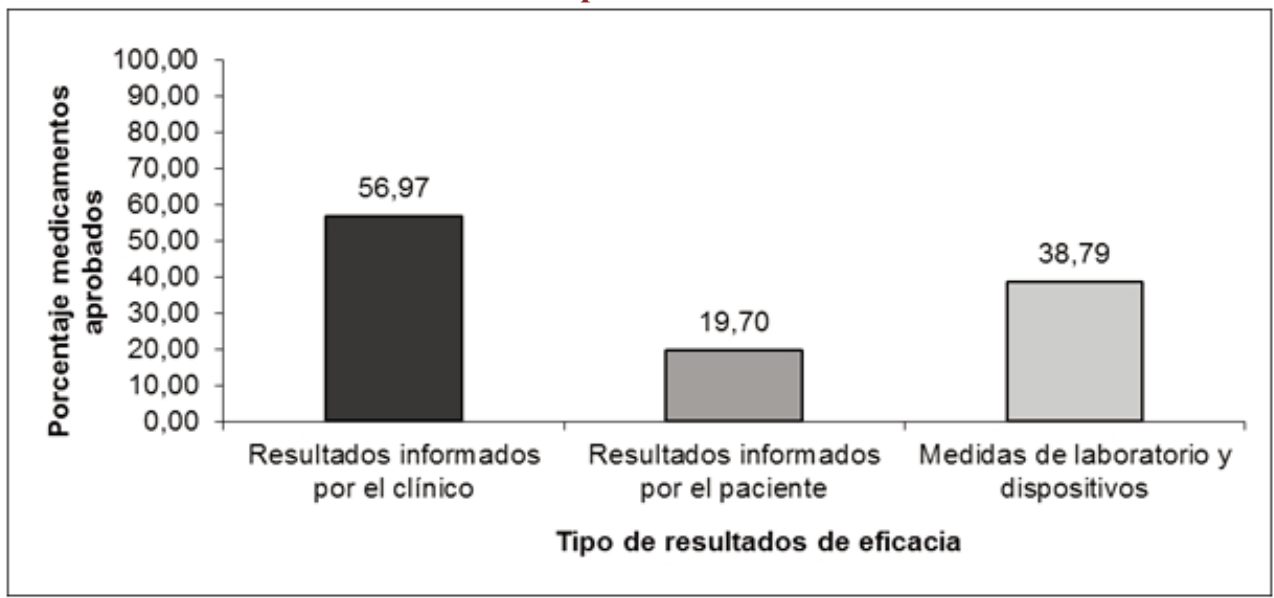


articulaciones inflamadas, evaluación global del clínico (escala de puntos de 0 a 5) y evaluación global de paciente (escala de puntos de 0 a 5). Igualmente, se contabilizaron todos los parámetros que componen el criterio de respuesta de forma individual.

La figura 1 muestra la distribución de resultados finales incluidos en fichas técnicas. Los CROs fueron los resultados finales de eficacia más utilizados, apareciendo en 188 (57\%) fichas técnicas de medicamentos $\left(\mathrm{chi}^{2}=96,85, \mathrm{p}<0,001\right)$. Las medidas de laboratorio o medidas realizadas mediante dispositivos (ML) aparecieron en $128(38,7 \%)$ fichas técnicas y se registraron PROs como resultados finales de eficacia en $65(19,7 \%)$ fichas técnicas de medicamentos. El $61,5 \%$ de los medicamentos recogieron un único tipo de resultado final de eficacia en sus fichas técnicas. Los CRO constaron en ficha técnica como único resultado final de eficacia en el 33,9\% de los medicamentos, las medidas de laboratorio o medidas realizadas mediante dispositivos en el $23,6 \%$ de los medicamentos y los
PRO únicamente en el 4,2\% de los medicamentos, siendo los CRO los resultados que más se utilizaron como resultado único $\left(\mathrm{chi}^{2}=58,0 ; \mathrm{p}<0,001\right)$. Respecto a la combinación de diversos tipos de resultados de eficacia, la utilización de CRO con PRO fue la más común apareciendo en el $9,7 \%$ de las fichas técnicas estudiadas, seguida de resultados de laboratorio con CRO, presente en el 9,1\% de las fichas técnicas. La utilización de los tres tipos de resultados finales considerados se dio en el $4,2 \%$ de los medicamentos, mientras que la utilización de medidas de laboratorio con PRO fue la combinación minoritaria con una proporción del 1,5\% (figura 2). En 188 medicamentos se incluyeron resultados CRO, de los que en 112 $(59,6 \%)$ (IC:52,6\%-66,6\%) el resultado CRO fue el único resultado incluido en la ficha técnica. Una proporción similar fue observada en los 128 medicamentos que incluyeron medidas de laboratorio, de los que en 78 casos $(60,9 \%)$ (IC:52,5\%$69,4 \%$ ) fue el único resultado comunicado. Estos hallazgos contrastan con el número de medicamentos con PROs. Un total de 65 medicamentos, de los que en

Figura 2

Tipos de variables de eficacia utilizadas en los ensayos clínicos descritos en las fichas técnicas de los medicamentos aprobados en España entre los años 200 y 2012 y sus asociaciones

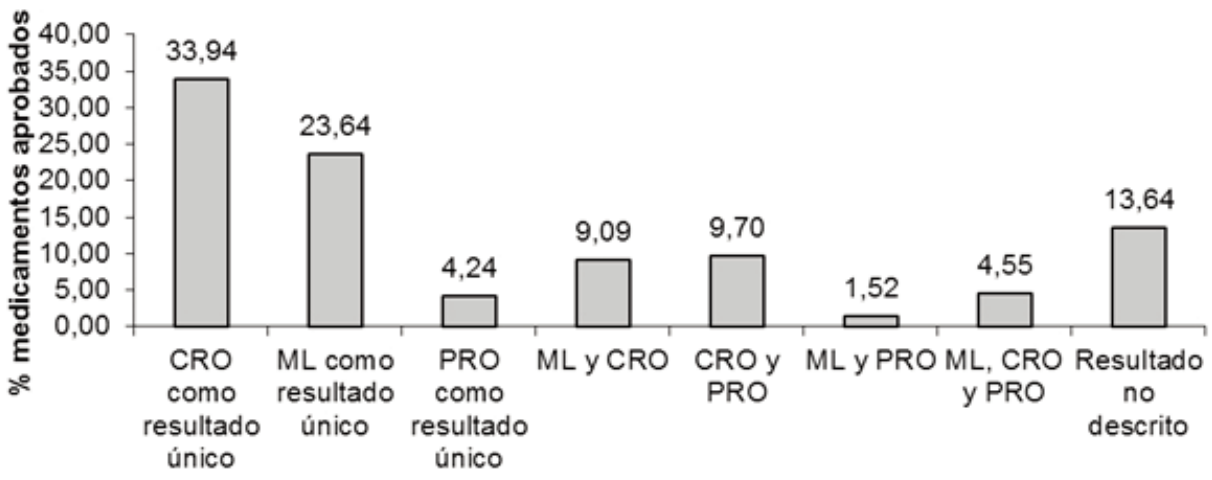

Tipo de asociación resultados

PRO $=$ Patient-reported-outcomes o resultados en salud comunicados por el paciente.

$\mathrm{CRO}=$ Clinical-reported outcomes o resultados comunicados por el médico

$\mathrm{ML}=$ Pruebas de laboratorio y dispositivos de medida 
Tabla 1

Distribución (\%) de resultados finales de eficacia por grupos terapéuticos

\begin{tabular}{|c|c|c|c|c|}
\hline \multirow[t]{2}{*}{ Clase terapéutica } & \multirow[t]{2}{*}{$\begin{array}{l}\mathrm{N}^{\circ} \text { total de } \\
\text { medicamentos } \\
\text { aprobados }\end{array}$} & \multicolumn{3}{|c|}{$\begin{array}{l}\text { Porcentaje de medicamen- } \\
\text { tos por tipo de } \\
\text { resultado final }\end{array}$} \\
\hline & & PRO & CRO & ML \\
\hline \begin{tabular}{|l} 
A. Sistema digestivo y metabolismo \\
\end{tabular} & 37 & 5,41 & 32,43 & 72,97 \\
\hline Diabetes & 14 & 0,00 & 7,14 & 100,00 \\
\hline Antieméticos & 3 & 0,00 & 100,00 & 0,00 \\
\hline Déficit enzimático o proteína & 12 & 8,33 & 41,67 & 83,33 \\
\hline B. Sangre y órganos hematopoyéticos & 24 & 4,17 & 66,67 & 29,17 \\
\hline Anemia & 4 & 0,00 & 75,00 & 100,00 \\
\hline Prevención tromboembolismo & 5 & 0,00 & 80,00 & 0,00 \\
\hline Prevención eventos aterotrombóticos & 3 & 0,00 & 100,00 & 0,00 \\
\hline C. Sistema cardiovascular & 23 & 4,35 & 43,48 & 65,22 \\
\hline Hipertensión arterial & 7 & 0,00 & 0,00 & 85,71 \\
\hline Conversión fibrilación a ritmo sinusal & 5 & 0,00 & 80,00 & 60,00 \\
\hline D. Medicamentos dermatologicos & 9 & 22,22 & 77,78 & 11,11 \\
\hline G. Genitourinario y hormonas & 11 & 63,64 & 54,55 & 27,27 \\
\hline H. Hormonas excluídas las sexuales & 10 & 0,00 & 40,00 & 70,00 \\
\hline J. Antiinfecciosos de uso sistemico & 33 & 0,00 & 42,42 & 57,58 \\
\hline Virus VIH & 12 & 0,00 & 0,00 & 100,00 \\
\hline Hepatitis B & 3 & 0,00 & 66,67 & 100,00 \\
\hline Neumonía & 6 & 0,00 & 50,00 & 0,00 \\
\hline Candidiasis & 4 & 0,00 & 100,00 & 25,00 \\
\hline L. Agentes antineoplasicos e inmunomoduladores & 72 & 25,00 & 88,89 & 29,17 \\
\hline Artritis reumatoide & 8 & 87,50 & 100,00 & 75,00 \\
\hline Artritis psoriásica & 3 & 66,67 & 100,00 & 66,67 \\
\hline Leucemia linfocítica crónica & 3 & 0,00 & 100,00 & 0,00 \\
\hline Leucemia linfoblástica aguda & 4 & 0,00 & 100,00 & 25,00 \\
\hline Cáncer colon-recto & 4 & 25,00 & 100,00 & 0,00 \\
\hline Profilaxis rechazo transplante & 4 & 0,00 & 75,00 & 0,00 \\
\hline Artritis idiopática juvenil & 4 & 100,00 & 100,00 & 100,00 \\
\hline Psoriasis & 3 & 66,67 & 100,00 & 0,00 \\
\hline Cáncer mama & 6 & 0,00 & 100,00 & 0,00 \\
\hline Leucemia mieloide crónica & 3 & 0,00 & 100,00 & 66,67 \\
\hline Mieloma múltiple & 4 & 0,00 & 100,00 & 50,00 \\
\hline Carcinoma células renales & 5 & 40,00 & 100,00 & 0,00 \\
\hline M. Sistema musculoesqueletico & 12 & 50,00 & 58,33 & 41,67 \\
\hline Alivio sintomático artrosis & 4 & 50,00 & 25,00 & 0,00 \\
\hline N. Sistema nervioso & 38 & 52,63 & 68,42 & 5,26 \\
\hline \begin{tabular}{|l|} 
Esquizofrenia \\
\end{tabular} & 5 & 60,00 & 80,00 & 0,00 \\
\hline Depresión & 3 & 66,67 & 100,00 & 0,00 \\
\hline Epilepsia & 6 & 16,67 & 100,00 & 0,00 \\
\hline P. Antiparasitarios & 4 & 0,00 & 0,00 & 50,00 \\
\hline R. Sistema respiratorio & 11 & 54,55 & 72,73 & 45,45 \\
\hline Rinitis estacional y alérgica & 4 & 100,00 & 75,00 & 0,00 \\
\hline Enf. obstructiva crónica EPOC & 4 & 75,00 & 50,00 & 100,00 \\
\hline S. Organos de los sentidos & 14 & 14,29 & 42,86 & 42,86 \\
\hline Glaucoma, hipertensión ocular & 5 & 0,00 & 0,00 & 100,00 \\
\hline Degeneración macular asociada a la edad & 4 & 50,00 & 100,00 & 0,00 \\
\hline V. Varios & 27 & 0,00 & 29,63 & 25,93 \\
\hline
\end{tabular}


Tabla 2

Descripción de resultados comunicados por el paciente según grupo terapéutico e indicación

\begin{tabular}{|c|c|c|}
\hline Grupo terapéutico & Indicación & PRO utilizado \\
\hline $\begin{array}{l}\text { A } \\
\text { Sistema digestivo } \\
\text { y metabolismo }\end{array}$ & Enfermedad de Fabry & $\begin{array}{l}\text { Escala validada de para la medición del dolor. } \\
\text { Instrumentos validados de medición de calidad de vida }\end{array}$ \\
\hline $\begin{array}{c}\text { B } \\
\text { Sangre } \\
\text { y órganos } \\
\text { hematopoyéticos }\end{array}$ & $\begin{array}{l}\text { Tratamiento sintomático de crisis agu- } \\
\text { das de angioedema hereditario (AEH) } \\
\text { en adultos }\end{array}$ & $\begin{array}{l}\text { Escala analógica visual (EAV) para la medición del ali- } \\
\text { vio de los síntomas. }\end{array}$ \\
\hline $\begin{array}{c}\text { C } \\
\text { Sistema } \\
\text { cardiovascular }\end{array}$ & $\begin{array}{l}\text { Hipertensión arterial pulmonar (HAP) } \\
\text { en adultos }\end{array}$ & $\begin{array}{l}\text { Escala de función física de la encuesta de SF-36 He- } \\
\text { alth Survey } \\
\text { Escala de Disnea de Borg (BDI) }\end{array}$ \\
\hline \multirow{2}{*}{$\begin{array}{l}\text { D } \\
\text { Medicamentos } \\
\text { dermatológicos }\end{array}$} & Hirtusismo facial en mujeres & $\begin{array}{l}\text { Escala visual analógica para la medición de malestar } \\
\text { psicológico con la enfermedad }\end{array}$ \\
\hline & $\begin{array}{l}\text { Tratamiento cutáneo de la queratosis } \\
\text { actínica no hiperqueratósica y no hi- } \\
\text { pertrófica en adultos }\end{array}$ & $\begin{array}{l}\text { Cuestionario de Satisfacción con el Tratamiento para } \\
\text { Medicamentos (TSQM) }\end{array}$ \\
\hline \multirow{4}{*}{$\begin{array}{c}\mathbf{G} \\
\text { Aparato } \\
\text { genitourinario } \\
\text { y hormonas sexuales }\end{array}$} & Hiperplasia benigna de próstata & $\begin{array}{l}\text { İndice de síntomas de la Asociación Urológica Ame- } \\
\text { ricana (AUA-SI) }\end{array}$ \\
\hline & Disfunción eréctil & $\begin{array}{l}\text { Tentativa de coitos satisfactorios Dominio IIEF-EF } \\
\text { (International Index of Erectile Function-Erectile } \\
\text { Function Domain) }\end{array}$ \\
\hline & Eyaculación precoz & Tiempo de latencia eyaculatoria intravaginal \\
\hline & Incontinencia urinaria & $\begin{array}{l}\text { Número de micciones diarias } \\
\text { Escalas de beneficio de tratamiento } \\
\text { Medidas de calidad de vida }\end{array}$ \\
\hline \multirow{11}{*}{$\begin{array}{c}\mathbf{L} \\
\text { Agentes } \\
\text { antineoplásicos } \\
\text { e } \\
\text { inmunomoduladores }\end{array}$} & $\begin{array}{l}\text { Artritis reumatoide, artritis idiopática } \\
\text { juvenil, espondilitis anquilosante, ar- } \\
\text { tritis psoriásica, psoriasis o enferme- } \\
\text { dad de Crohn }\end{array}$ & $\begin{array}{l}\text { Criterios del Colegio Americano de Reumatología } \\
\text { (ACR) }\end{array}$ \\
\hline & Mesotelioma pleural maligno & $\begin{array}{l}\text { Escala Síntomas de Cáncer de Pulmón (Lung Cancer } \\
\text { Symptom Scale LCSS) }\end{array}$ \\
\hline & $\begin{array}{l}\text { Carcinoma metastático de colon o } \\
\text { recto }\end{array}$ & $\begin{array}{l}\text { Escala ECOG (Eastern Cooperative Oncology } \\
\text { Group) para calidad de vida }\end{array}$ \\
\hline & Cáncer medular de tiroides (CMT) & Brief Pain Inventory (BPI) \\
\hline & Espondilitis anquilosante & $\begin{array}{l}\text { Bath Ankylosing Spondylitis Functional Index: } \\
\text { BASFI de funcionamiento físico }\end{array}$ \\
\hline & Artritis reumatoide & $\begin{array}{l}\text { Indice de discapacidad del Cuestionario de Evalua- } \\
\text { ción del Estado de Salud HAQ (Health Assessment } \\
\text { Questionnaire), Cuestionario de Salud Abreviado SF } \\
36 \text { (Short Form Health Survey) } \\
\text { Escala de valoración funcional del tratamiento de } \\
\text { enfermedades crónicas FACIT (Functional Assess- } \\
\text { ment of Chronic Illness Therapy) }\end{array}$ \\
\hline & Enfermedad Crohn pediátrica & $\begin{array}{l}\text { Índice de actividad de la enfermedad de Crohn pe- } \\
\text { diátrica PCDAI }\end{array}$ \\
\hline & Enfermedad Crohn & $\begin{array}{l}\text { Indice de Actividad de la Enfermedad de Crohn } \\
\text { (CDAI) } \\
\text { Cuestionario de la Enfermedad Inflamatoria Intesti- } \\
\text { nal (IBDQ) }\end{array}$ \\
\hline & Gota artrítica & Escala analógica visual de dolor \\
\hline & $\begin{array}{l}\text { Cáncer de pulmón no microcítico } \\
\text { avanzado o metastásico }\end{array}$ & $\begin{array}{l}\text { FACT-L (Functional assessment of cancer therapy } \\
\text { lung) } \\
\text { Lung Cancer Symptom Scale LCSS }\end{array}$ \\
\hline & $\begin{array}{l}\text { Tratamiento de la esplenomegalia en } \\
\text { pacientes adultos con mielofibrosis } \\
\text { primaria, mielofibrosis secundaria a } \\
\text { policitemia vera o mielofibrosis se- } \\
\text { cundaria a trombocitemia esencial. }\end{array}$ & $\begin{array}{l}\text { Formulario de Evaluación de los Síntomas de Mielo- } \\
\text { fibrosis (MFSAF) }\end{array}$ \\
\hline
\end{tabular}


Tabla 2

(continuación)

\begin{tabular}{|c|c|c|}
\hline Grupo terapéutico & Indicación & PRO utilizado \\
\hline \multirow{4}{*}{$\begin{array}{c}\mathbf{L} \\
\text { Agentes } \\
\text { antineoplásicos } \\
\mathrm{e} \\
\text { inmunomoduladores }\end{array}$} & $\begin{array}{l}\text { Psoriasis en placas, crónica, } \\
\text { moderada o grave }\end{array}$ & $\begin{array}{l}\text { Índice de Calidad de Vida en Dermatología (DLQI) } \\
\text { Evaluación de los Síntomas de Psoriasis (PSA) } \\
\text { Componentes físico y mental del SF-36 } \\
\text { Escala de Ansiedad y Depresión en el Hospital (HADS) } \\
\text { Cuestionario sobre Limitaciones Laborales (WLQ) }\end{array}$ \\
\hline & $\begin{array}{l}\text { Tumores neuroendocrinos } \\
\text { pancreáticos }\end{array}$ & $\begin{array}{l}\text { Cuestionario de Calidad de Vida de la Organización } \\
\text { Europea para la Investigación y el Tratamiento del } \\
\text { Cáncer (EORTC QLQC-30) }\end{array}$ \\
\hline & Carcinoma células renales & Supervivencia ajustada por calidad vida \\
\hline & Cáncer próstata metastásico & $\begin{array}{l}\text { Escala ECOG (Eastern Cooperative Oncology Group) } \\
\text { Cuestionario BPI-SF (Brief Pain Inventory-Short } \\
\text { Form) }\end{array}$ \\
\hline \multirow{6}{*}{$\begin{array}{c}\mathbf{M} \\
\text { Sistema } \\
\text { musculoesquelético }\end{array}$} & $\begin{array}{l}\text { Alivio sintomático de la artrosis, la artri- } \\
\text { tis reumatoide (AR), la espondilitis an- } \\
\text { quilosante y el dolor y signos de infla- } \\
\text { mación asociados a la artritis gotosa } \\
\text { aguda. }\end{array}$ & $\begin{array}{l}\text { Mejoría de dolor y del estado de la enfermedad eva- } \\
\text { luado por el paciente }\end{array}$ \\
\hline & $\begin{array}{l}\text { Fusión vertebral lumbar } \\
\text { anterior a nivel de L4-S1, en adultos } \\
\text { con enfermedad de disco degenerativa }\end{array}$ & Escala Oswestry para dolor y discapacidad \\
\hline & Distonía cervical (tortícolis) & $\begin{array}{l}\text { Escala TWSTRS (Toronto Western Spasmodic Torti- } \\
\text { collis Rating Scale) } \\
\text { Escala visual analogical evaluación global paciente }\end{array}$ \\
\hline & $\begin{array}{l}\text { Tratamiento de la seudoartrosis tibial } \\
\text { cuando autoinjerto ha fracasado }\end{array}$ & $\begin{array}{l}\text { Dolor y capacidad de soportar peso comparado con au- } \\
\text { toinjerto }\end{array}$ \\
\hline & $\begin{array}{l}\text { Alivio sintomático en el tratamiento de } \\
\text { la artrosis de rodilla y cadera. }\end{array}$ & $\begin{array}{l}\text { Dolor, la rigidez, la función y la valoración del esta- } \\
\text { do de la enfermedad por parte del paciente }\end{array}$ \\
\hline & Osteoporosis & $\begin{array}{l}\text { Escala específica QUALIOST } \\
\text { Îndice de percepción de salud general de la escala } \\
\text { general SF-36 }\end{array}$ \\
\hline \multirow{6}{*}{$\begin{array}{l}\mathbf{N} \\
\text { Sistema } \\
\text { nervioso }\end{array}$} & Esquizofrenia & $\begin{array}{l}\text { Escala PANNS } \\
\text { MADRS (Montgomery Asberg Depression Rating } \\
\text { Scale) } \\
\text { Escala de Impresión Clínica Global de gravedad de } \\
\text { la enfermedad en manía (ICG-BP), Aberrant Beha- } \\
\text { viour Checklist Irritability (ABC-I) } \\
\text { Escala de Rendimiento Personal y Social (PSP) }\end{array}$ \\
\hline & Depresión & $\begin{array}{l}\text { Escala de Hamilton para la evaluación de la depre- } \\
\text { sión (Hamilton Depression Rating Scale, HAM-D) }\end{array}$ \\
\hline & Enfermedad de Alzheimer & $\begin{array}{l}\text { ADCS-ADL severe (Modified Alzheimer's Disease } \\
\text { Cooperative Study Activities of Daily Living inven- } \\
\text { tory for Severe Alzheimer's Disease) para la evalua- } \\
\text { ción de la funcionalidad del paciente } \\
\text { Severe Impairment Battery (SIB) para evaluación de } \\
\text { síntomas } \\
\text { ADAS-cog (Alzheimer's Disease Assessment Scale, } \\
\text { Cognitive part) para la evaluación del funcionamien- } \\
\text { to psicológico del paciente }\end{array}$ \\
\hline & Enfermedad de Parkinson & $\begin{array}{l}\text { Escala de evaluación de la enfermedad de Parkinson } \\
\text { unificada (UPDRS) }\end{array}$ \\
\hline & $\begin{array}{l}\text { Esclerosis múltiple con } \\
\text { discapacidad en la marcha }\end{array}$ & $\begin{array}{l}\text { Escala } 12 \text { ítems de la marcha de esclerosis múltiple } \\
\text { (MSWS). }\end{array}$ \\
\hline & Síndrome de Piernas Inquietas idiopático & $\begin{array}{l}\text { Escala Internacional del Síndrome de Piernas } \\
\text { Inquietas (IRLS) }\end{array}$ \\
\hline
\end{tabular}


Tabla 2

(continuación)

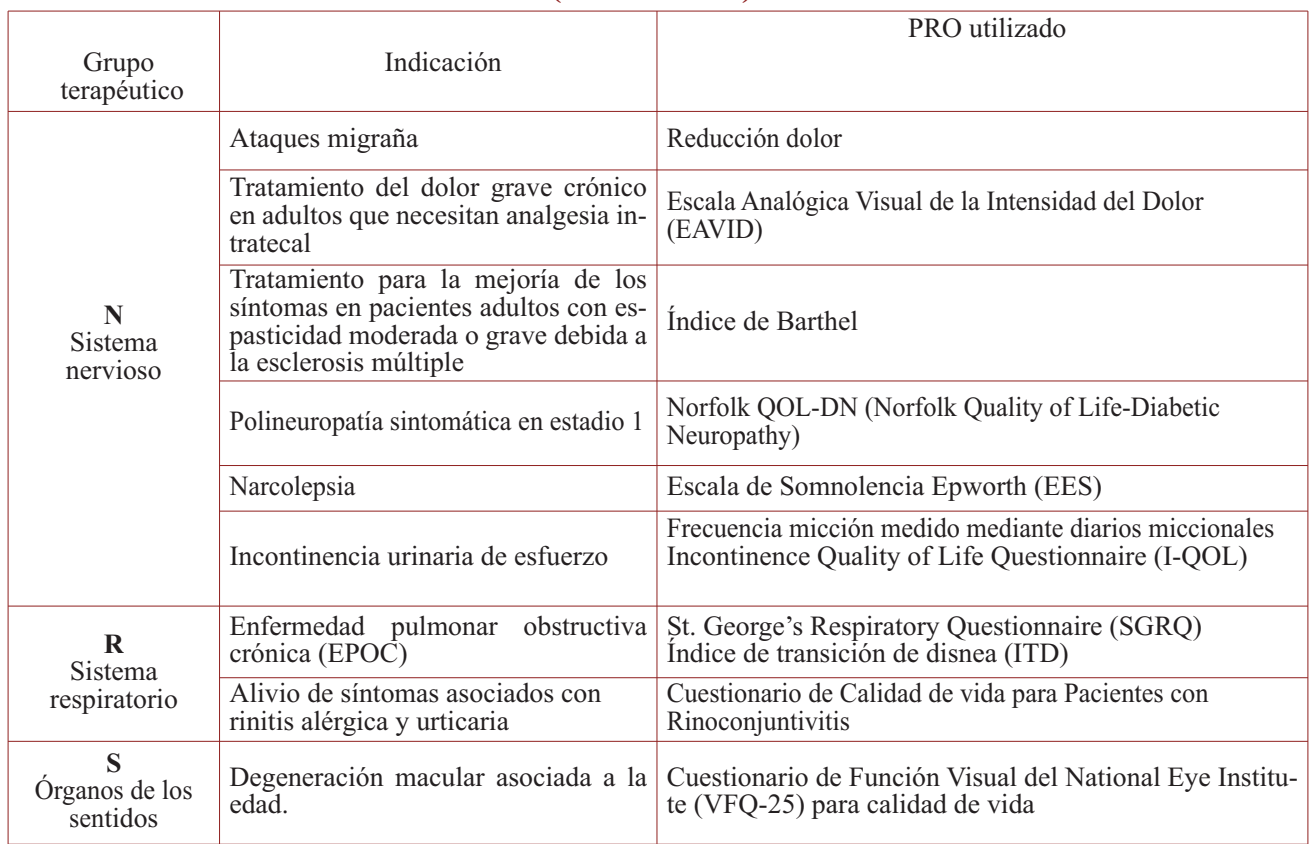

\section{Figura 3}

\section{Número y porcentaje de medicamentos con PROs en ficha técnica autorizados por año} durante el periodo de estudio con análisis de tendencia lineal en el periodo analizado

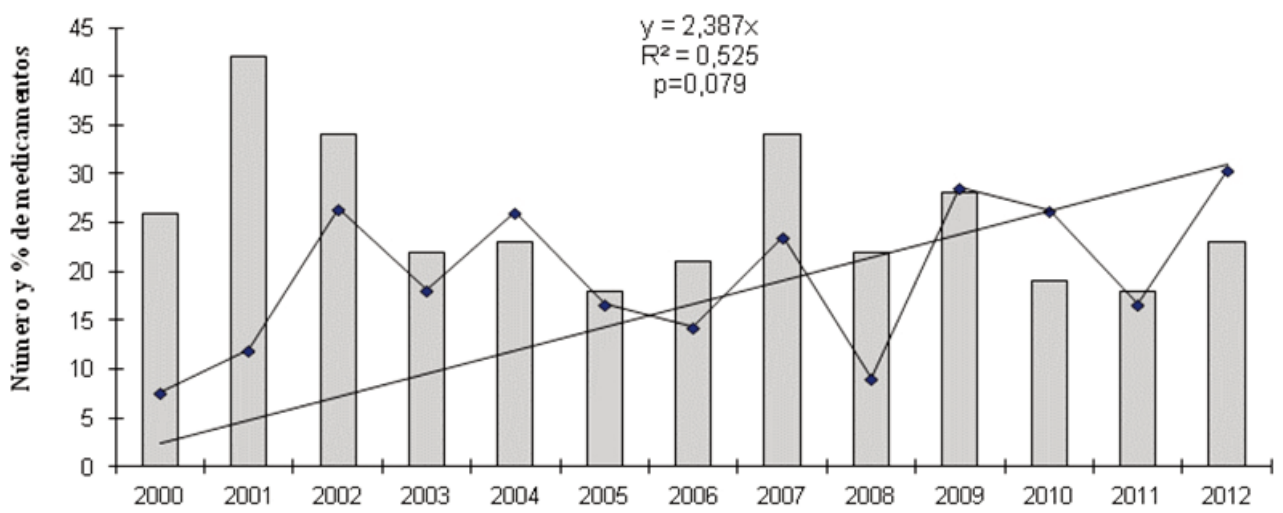

Barras representan número de medicamentos y línea con marcadores indica el porcentaje de medicamentos con PRO en la ficha técnica 
solo 14 (21,5\%) (IC:11,5\%-31,5\%) los resultados PRO fueron los únicos incluidos en la ficha técnica. Respectivamente, la probabilidad de incluir solo un resultado PRO en la ficha técnica cuando se incluyeron resultados PRO fue cercana al $80 \%$ inferior, tanto con respecto a resultados CRO [odds ratio $=0,19$ (IC:0,10$0,36), p<0,001]$, como frente a resultados ML. Odds ratio=0,18 (IC:0,09-0,35), $\mathrm{p}<0,001$. En el caso de PROs, se utilizaron casi cuatro veces más en combinación con otros tipos de resultados $(78,46 \%)$ que como resultado único $(21,53 \%)$, siendo la asociación más frecuente con CROs $(49,2 \%)$.

Hubo una gran variación en el uso de resultados finales por clase terapéutica. Los grupos terapéuticos con más utilización de PROs en ficha técnica fueron los medicamentos para el sistema genitourinario $(63,3 \%$ de los medicamentos del grupo presentan PRO), para el sistema respiratorio $(54,5 \%)$, para el sistema nervioso $(52,6 \%)$, para el sistema musculoesquelético $(50 \%)$ y agentes antineoplásicos e inmunomoduladores $(25 \%)$. La tabla 1 muestra la frecuencia de resultados de eficacia por grupo terapéutico, describiendo en cada grupo las indicaciones para los cuales existía un mínimo de tres medica- mentos aprobados. No se tuvieron en cuenta los medicamentos para los que no se encontró ficha técnica o bien no se especificó el grupo en ella. Fueron 5 medicamentos y sólo 1 de ellos expresó su resultado final de eficacia mediante ML.

Respecto a la utilización de PROs, la tabla 2 muestra los resultados comunicados por el paciente que fueron considerados más relevantes clasificados según el grupo terapéutico y la indicación terapéutica. La figura 3 muestra el porcentaje de medicamentos autorizados con PROs en sus fichas técnicas frente al total de medicamentos autorizados por año. En los años 2000, 2001 y 2008 el porcentaje de medicamentos con PROs fue inferior al 10\%, mientras que en los restantes 10 años este porcentaje rondó o fue superior al 20\%. El análisis de tendencia lineal mostró una relación lineal creciente con orientación hacia la significación estadística: $R 2=0,525, p=0,079$ (figura 3 ).

Durante la revisión de las fichas técnicas se encontraron diferentes tipos de medidas de PROs. La figura 4 muestra la frecuencia con la se encontraron, destacando las medidas de síntomas $(70,77 \%$ de medicamentos con PRO) y la medición de calidad de vida relacionada con la salud $(60 \%)$ como los más utilizados.

Figura 4

Distribución (\%) de las medidas de PROs en el estudio

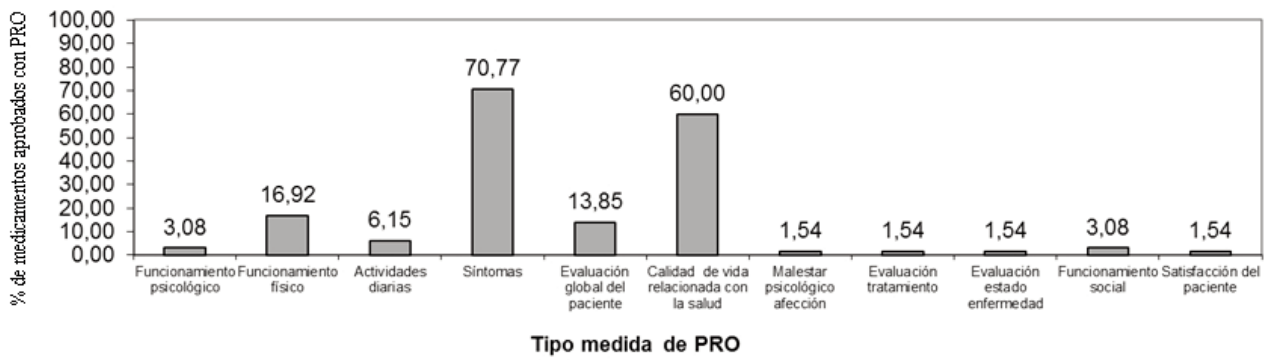

PRO = Patient-reported-outcomes o resultados en salud comunicados por el paciente 


\section{DISCUSIÓN}

Según los resultados del estudio, la inclusión de PROs en fichas técnicas es aún minoritaria frente a otros tipos de resultados, como las mediciones de laboratorio o los resultados informados por el médico y todavía es menor la utilización de PROs como resultado único final de eficacia, aunque en algunas áreas terapéuticas sí son habituales, como sistema nervioso, antineoplásicos e inmunomoduladores, sistema genitourinario y sistema respiratorio. Es esperable que la utilización de PROs como resultados finales en ensayos clínicos sea más común en el desarrollo de los medicamentos a medida que su uso y evaluación sean más cotidianos dentro de la práctica clínica, como parece atestiguar el moderado incremento de referencias a PROs observados en las fichas técnicas a lo largo del periodo de tiempo estudiado, aunque puede ser debido al escaso número de fichas técnicas conteniendo PROs en los primeros años del periodo evaluado. El análisis de la utilización de los diferentes tipos de resultados de eficacia estudiados en los diversos grupos terapéuticos muestra la práctica clínica habitual en el diagnóstico y valoración de las diferentes patologías. Para enfermedades como la artritis reumatoide, enfermedad pulmonar obstructiva crónica (EPOC), disfunción eréctil, enfermedad de Alzheimer, hiperplasia benigna de próstata o tratamiento de dolor, la información proporcionada por el paciente tiene un valor importante en la evaluación de la eficacia del tratamiento debido a la naturaleza de la patología. Tampoco es extraño que para enfermedades como el síndrome de inmunodeficiencia adquirida, hepatitis, enfermedades infecciosas, déficits enzimáticos o hipertensión ocular, se confíe más en la utilización de medidas realizadas en el laboratorio o valoraciones del médico para expresar su eficacia. Sin embargo, sí es destacable que para enfermedades crónicas que se supone tienen un alto impacto en la calidad de vida del paciente, como la hipertensión arterial o la diabetes $^{21,22,}$ no se utilice la información proporcionada por el paciente para complementar otros resultados de eficacia. También llama la atención el escaso uso de PRO en medicamentos para el cáncer, que es un problema de salud crónico en el que se esperaría el uso de PROs de calidad de vida y/o funcionamiento del paciente. Esto puede ser debido a preferencias del médico por otros tipos de resultados, una escasa valoración de la utilidad de los PROs, falta de instrumentos apropiados de medición en la población del ensayo clínico o una falta de apoyo regulatorio por parte de las autoridades para la generalización del uso de PROs.

Dentro de los diferentes tipos de PROs, los más utilizados fueron los instrumentos para la evaluación de los síntomas percibidos por el paciente de su enfermedad. Una posible interpretación es que la EMA acepta estos resultados como primarios o secundarios en ensayos clínicos utilizados en el registro del medicamento ${ }^{1,2}$. No obstante, aunque la EMA se refiere a la evaluación de la CVRS como un resultado opcional y que la base para la aprobación de un medicamento es su seguridad y eficacia en una indicación dada, el segundo tipo de resultado informado por el paciente más utilizado fue la CVRS medida a través de instrumentos formales multiitem, revelando una alta valoración de estos instrumentos por parte de los clínicos y una amplia aceptación por parte de las agencias reguladoras, al menos en ciertas áreas terapéuticas.

El estudio presenta limitaciones, además de la temporal. No todas las fichas técnicas describen cómo se ha medido o registrado la información obtenida del paciente. En casos como mejoría de síntomas o reducción del dolor, aunque la información provenga del propio paciente, desconocemos si ha sido recogida directamente mediante algún instrumento formal o escala visual o si el clínico ha cumplimentado algún tipo de escala con información dada por el paciente para la valoración de dichos resultados. La progre- 
sión creciente observada en el uso de PROs, que mostró una tendencia hacia la significación estadística, puede estar enmascarada por el menor uso de PRO en los primeros años de análisis incluidos en el estudio y también porque el estudio incluyó un número limitado de años que, si se mantiene la tendencia, en un futuro podría confirmar la mencionada mayor inclusión de PROs en las fichas técnicas aprobadas en los últimos años.

Se han realizado estudios similares en Estados Unidos con el objetivo de estudiar la utilización de PROs como medidas de eficacia en el etiquetado de los medicamentos. Richard J. Willke y colaboradores ${ }^{18}$ estudiaron las medidas de eficacia utilizadas en nuevos medicamentos aprobados por la FDA desde 1997 a 2002. De un total de 215 moléculas aprobadas, el 30\% incluyeron PROs como medidas de eficacia en el etiquetado. Posteriormente, Ari Gnanasakthy y colaboradores ${ }^{23}$ realizaron el mismo estudio en medicamentos aprobados en Estados Unidos entre el año 2006 y 2010. En este período se aprobaron 116 nuevos medicamentos y el 24\% mostraron PROs como resultados finales de eficacia en su etiquetado.

Comparando los resultados, podemos concluir que la utilización de PROs como resultado de eficacia es mayor en países como Estados Unidos. Una posible explicación a este hecho es, como ya se ha planteado anteriormente, que la FDA parece conceder una mayor importancia a los PRO como resultado final de eficacia en ensayos clínicos que otras agencias reguladoras como la EMA.

Sin embargo, ambos estudios revelan que los medicamentos con más utilización de PROs son los medicamentos del sistema nervioso central y los medicamentos antiinflamatorios, reumatológicos y analgésicos. Estos resultados concuerdan con los obtenidos en este estudio, lo que corrobora que en ciertas patologías la información proporcionada por el paciente tiene un valor importante en la evaluación de la eficacia del tratamiento debido a su naturaleza.

En conclusión, a pesar de las limitaciones observadas, en este estudio parece observarse la existencia de una tendencia creciente en el uso de PROs en las fichas técnicas de medicamentos aprobados en España en el periodo comprendido entre los años 2000 a 2012, aunque no se puede concluir que sea una tendencia lineal, ya que los resultados no han mostrado significación estadística. Notablemente, los síntomas de enfermedad informados por el paciente y la calidad de vida relacionada con la salud son los PROs principalmente incluidos en las fichas técnicas. No obstante, la frecuencia de inclusión de los PROs sigue siendo claramente inferior al de otros resultados, como los comunicados por los clínicos o los de laboratorio. Finalmente, la probabilidad de que una ficha técnica incluya únicamente resultados PROs es significativamente inferior a que sólo se incluyan resultados de laboratorio o informados por el clínico.

\section{BIBLIOGRAFÍA}

1. European Medicines Agency. Committee for medicinal products for human use. Reflection paper on the regulatory guidance for the use of health-related quality of life (HRQL) measures in the evaluation of medicinal products. Doc. Ref. EMA/CHMP/EWP/139391/2004. London: EMA; 2005 .

2. Chassany O, Sagnier P, Marquis P, Fullerton S, Aaronson $\mathrm{N}$ for the European Regulatory Issues on Quality of Life Assessment Group. Patient-reported outcomes: the example of health-related quality of life A European guidance document for the improved integration of health-related quality of life assessment in the drug regulatory process. Drug Inf J. 2002; 36: 209-38

3. Guidance for industry. Patient-reported outcome measures: Use in medical product development to support labelling claims: draft guidance. Health Qual Life Outcomes. 2006; 4:79. 
4. Department of Health and Human Services Food and Drug Administration Guidance for Industry: Patient-Reported Outcome Measures: Use in Medical Product Development to Support Labeling Claims. U.S. FDA, Clinical/Medical. 2009. Disponible en: http://www.fda.gov.downloads/Drugs/Guidances/UC M193282.pdf

5. Garrat A. The role of patient reported outcomes in the regulatory process needs to be better defined. Instuto Superiore di Sanità. Response to editorial: Patient reported outcome measures in trials. BMJ. 2009; 338: a2597.

6. Ad hoc Committee on Medical Ethics American College of Physicians: American Colleague of Physicians Ethics Manual Part 1. Ann Intern Med. 1984, 101:129. 37.

7. Waterworth S, Luker KA: Reluctant Collaborators. Do patients want to be involved in decision concerning care? J Adv Nurse. 1990, 15:971-6.

8. Guadagnoli E, Ward P. Patient Participation in Decision Making Soc Sci Med. 1998, 47(3):329-39.

9. Twachtman G. Comparative Effectiveness Needs Holistic Value Measures - Amgen Exec. Disponible en: http://www.pharmamedtechbi.com/publications/the-pink-sheet/70/050/comparative-effectiveness-needs-holistic-value-measures--amgen-exec

10. Doward LC, Gnanasakthy A, Baker MG. Patient reported outcomes: looking beyond the label claim. Health Qual Life Outcomes. 2010; 8:89.

11. Hewlett SA. Patients and Clinicals Have Different Perspectives on Outcomes in Arthritis. J Rheumatol. 2003; 30:877-9.

12. Martin RL, Mohtadi NG, Safran MR, Leunig M, Martin HD, McCarthy J, Guanche CA, Kelly BT, Byrd JW Clohisy JC, Philippon MJ, Sekiya JK. Differences in Physical and Patient Ratings of Items Used to Assess Hip Disorders. Am J Sports Med. 2009; 37: 150812.

13. Wehmeier PM, Kluge M, Schacht A, Helsberg K, Schreiber W. Correlation of physician and patient rated quality of life during antipsychotic treatment in outpatients with schizophrenia. Schizophr Res. 2007; 91:178-86.

14. Patterson MA, Larsen H, Pedersen L, Sonne N, Groenvold M. Assessing Health-related quality of life in palliative care: comparing patient and physician assessments. Eur J Cancer. 2006; 42:1159-66.
15. Leong KP, Chong EY, Kong KO, Chan SP, Thong BY, Lian Ty, Chng HH, Koh ET, Tec CL, Lau TC, Law WG, Cheng YK, Badsha H, Chew LC, Yong WH, Howe HS, Tan Tock Seng Hospital (TTSH) Lupus Study Group. Discordant assessment of lupus activity between patients and their physicians: the Singapore experience. Lupus. 2010; 19: 100-6.

16. Srikrishna S, Robinson D, Cardozo L, González J. Is there a difference in patient and physician quality of life evaluation in pelvic organ prolapse? Int Urogynecol J. 2008; 19:517-20.

17. Yalcin I, Viktrup L. Comparison of physician and patient assessments of incontinence severity and improvement. Int Urogynecol J. 2007;11: 1291-5.

18. Willke RJ, Burke LB, Erickson P. Measuring treatment impact: a review of patient-reported outcomes and other efficacy endpoints in approved product labels. Control Clin Trials. 2004; 25:535-52.

19. Patrick DL, Erickson P. Health status and health policy: quality of life in health care evaluation and resource allocation. New York: Oxford University Press; 1993.

20. SPSSWIN program. Versión 17.0. SPSS Inc., Chicago, IL, USA.

21. Rubin RR, Peyrot M. Quality of life and diabetes. Diabetes/Metab Res Rev. 1999; 15: 205-18.

22. Hirsch A, Bartholomae C, Volmer T. Dimensions of quality of life in people with non insuline dependent diabetes. Qual Life Res. 2000; 9:207-18.

23. Gnanasakthy A, Mordin M, Clark M, DeMuro C, Fehnel S, Copley-Merriman C. A review of patient-reported outcome labels in the United States: 2006 to 2010. Value Health. 2012; 15:437-42. 